\title{
Accuracy assessment of open - pit mine's digital surface models generated using photos captured by Unmanned Aerial Vehicles in the post - processing kinematic mode
}

\author{
Long Quoc Nguyen* \\ Hanoi University of Mining and Geology, Hanoi, Vietnam
}

\begin{abstract}
ARTICLE INFO
ABSTRACT

Article history:

Received 19th Mar. 2021

Revised 23rd June 2021

Accepted $19^{\text {th }}$ July 2021

Keywords:

Digital Surface Model,

Ground control points,

Open-pit mine,

Unmanned aerial vehicle.

To evaluate the accuracy of the digital surface model (DSM) of an open-pit mine produced using photos captured by the unmanned aerial vehicle equipped with the post-processing dynamic satellite positioning technology (UAV/PPK), a DSM model of the Deo Nai open-pit coal mine was built in two cases: (1) only using images taken from UAV/PPK and (2) using images taken from UAV/PPK and ground control points (GCPS). These DSMs are evaluated in two ways: using checkpoints (CPS) and comparing the entire generated DSM with the DSM established by the electronic total station. The obtained results show that if using CPS, in case 1, the errors in horizontal and vertical dimension were 6.8 and $34.3 \mathrm{~cm}$, respectively. When using two or more GCPs (case 2), the horizontal and vertical errors are at the centimetre-level (4.5 cm and $4.7 \mathrm{~cm}$ ); if using the DSM comparison, the same accuracy as case 2 was also obtained.
\end{abstract}

Copyright (C) 2021 Hanoi University of Mining and Geology. All rights reserved.

${ }^{*}$ Corresponding author

E - mail: nguyenquoclong@humg.edu.vn

DOI: 10.46326/JMES.2021.62(4).05 


\title{
Tạp chí Khoa học Kỹ thuật Mỏ - Địa chất
}

Trang điện tử: http://tapchi.humg.edu.vn

\section{Đánh giá độ chính xác mô hình số bề mặt mỏ lộ thiên thành lập từ dữ liệu máy bay không người lái có định vị tâm chụp ảnh bằng công nghệ đo động xử lý sau}

\author{
Nguyễn Quốc Long* \\ Trường Đại học Mỏ - Địa chất, Hà Nội, Việt Nam
}

THÔNG TIN BÀI BÁO T TÓM TẮT

Quá trình:

Nhận bài 19/3/2021

Sưa xong 23/6/2021

Chap nhận đăng 19/7/2021

Tù̀ khóa:

Điểm khống chế mặt đất,

Máy bay không người lái,

Mỏ lộ thiên,

Mô hình số bề mặt.
Để đánh giá độ chính xác mô hình số bề mặt (DSM) mỏ lộ thiên thành lập tùr dũ liệu máy bay không người lái có định vị tâm chụp ảnh bằng công nghệ định vị vệ tinh động xử lý sau (UAV/PPK), nghiên cúu này đã tiến hành xây dựng mô hình DSM mỏ than Đèo Nai với 2 trường hợ: (1) chỉ sư dụng ảnh chụp tù̀ $U A V / P P K$ và (2) sư dưng ảnh chup tù̀ UAV/PPK kết hợp với các điểm khống chế mặt đất (GCP). Các DSM được đánh giá độ chính xác bằng 2 phưong pháp là so sánh các điểm trên DSM với các điểm kiểm tra (CP) tương úng trên bề mặt mỏ và so sánh toàn bộ DSM được tạo ra với DSM thành lập bằng máy toàn đạc điện tử. Kết quả nhận được cho thấy: nếu sủ dụng CP, trường hơp 1 cho sai số về mặt bằng là $6,8 \mathrm{~cm}$ và độ cao là $34,3 \mathrm{~cm}$. Trường hợp 2 khi kết hợp với 2 điểm khống chế ảnh trở lên thì sai số cả mặt bằng và độ cao đạt cỡ centi-mét (4,5 cm và 4,7 cm); nếu sủ dụng cách đánh giá thú 2 là so sánh trực tiếp DSM tùu UAV với DSM do mỏ than Đèo Nai thành lập bằng máy toàn đạc điện tử thì cũng cho độ chính xác tương đồng với truờng hợp 2.

\section{Mở đầu}

Tại các mỏ lộ thiên khai thác than ở Quảng Ninh, đo vẽ bản đồ địa hình được thực hiện thường xuyên nhằm cập nhật hiện trạng mỏ phục vụ quản lý, tính khối lượng, thiết kế khai thác, đảm bảo an toàn,... Hiện nay, đo vẽ bản đồ tại các mỏ chủ yếu được thực hiện bằng thiết bị toàn đạc điện tử, phương pháp này tốn nhiều thời gian và công sức do địa hình mỏ biến đổi liên tục (Nguyễn Quốc

\footnotetext{
*Tác giả liên hệ

E - mail: nguyenquoclong@humg.edu.vn DOI: 10.46326/JMES.2021.62(4).05
}

Long và Lê Văn Cảnh, 2020). Một số công nghệ mới đã được quan tâm nghiên cứu trong lĩnh đo đạc bản đồ ở mỏ như công nghệ định vị vệ tinh sử dụng hệ thống mạng lưới trạm tham chiếu hoạt động liên tục (GNSS/CORS) (Nguyen Viet Nghia và nnk., 2016), quét laser mặt đất (TLS) (Nguyen Quoc Long và nnk., 2018; Nguyen Viet Nghia và nnk., 2019), thiết bị bay không người lái (UAV) (Nguyễn Viết Nghĩa, 2020). Trong đó, UAV là công nghệ được quan tâm nhất hiện nay do giá thành thấp hơn so với công nghệ quét laser, hơn nữa thời gian đo đạc ngắn, hiệu suất lao động cao, tiếp cận được các vùng địa hình khó khăn (Bui T. D. và nnk., 2017; Nguyen Q. L. và nnk., 2019). 
Công nghệ này cũng được chứng minh đáp ứng được độ chính xác khi thành lập bản đồ địa hình tỷ lệ lớn ở các mỏ khai thác than và vật liệu xây dựng ở Việt Nam. (Nguyen Q. L. và nnk., 2020; Nguyen Q. L. và nnk., 2021; Le V.C. và nnk., 2020). Nhược điểm lớn nhất khi ứng dụng công nghệ này là phải thành lập nhiều điểm điểm khống chế ảnh mặt đất (GCP). Đây là công việc chiếm đa số thời gian ngoại nghiệp (Forlani và nnk., 2018), đặc biệt đối với địa hình phức tạp như ở mỏ lộ thiên, nhược điểm này càng thể hiện rõ.

Nghiên cứu tích hợp công nghệ định vị vệ tinh động tức thời (GNSS/RTK) lên UAV nhằm nâng cao độ chính xác định vị tâm chụp ảnh, giảm thiểu số lượng điểm GCP đã được nhắc đến trong một số nghiên cứu. Fazeli và cộng sự đã gắn máy thu GNSS lên UAV và tiến hành bay chụp thực nghiệm. Kết quả cho thấy, độ chính xác của DSM vẫn chỉ ở mức đề-xi-mét mặc dù số lượng điểm GCP gần như tương đương với khi sử dụng UAV thường (Fazeli và nnk., 2016). Nghiên cứu của Fazeli cũng chỉ ra sai số này là do tồn tại sự trễ pha giữa thời điểm chụp ảnh của camera và thời điểm xác định tâm chụp ảnh của thiết bị đo động tức thời (RTK) trên máy bay. Vấn đề này đã dần được khắc phục bởi các hãng sản xuất các thiết bị UAV/RTK, ví dụ như Phantom 4 RTK của hãng DJI có độ trễ giữa chụp ảnh và đo RTK chỉ ở mức mm (DJI, 2020). Một nghiên cứu khác được công bố bởi tác giả Zhang và cộng sự đã chứng minh định vị tâm chụp ảnh theo phương thức đo động tức thời GNSS/RTK cho độ chính xác đến xen-ti-mét (Zhang và nnk., 2019). Tác giả Taddia và cộng sự đã tiến hành khảo sát độ chính xác của thiết bị này khi ứng dụng trong thành lập bản đồ địa hình ven biển, kết quả khẳng định rằng UAV/RTK cho phép đo đạc đạt độ chính xác địa hình đến xen-ti-mét (Taddia và nnk., 2019). Tiến hành bay chụp địa hình vùng đồng bằng để khảo sát số lượng điểm GCP cần thiết khi sử dụng UAV/RTK được thực hiện trong các nghiên cứu của tác giả Forlani và các cộng sự. Nghiên cứu của họ chỉ ra rằng khi không sử dụng GCP thì DSM thành lập được có sai số lớn, nhưng khi kết hợp với chỉ 01 điểm GCP cho DSM có độ chính xác cao hơn rất nhiều (Forlani và nnk., 2018).

Từ các kết quả nghiên cứu trên thế giới ở trên có thể thấy rằng: đánh giá khả năng ứng dụng cũng như khảo sát số lượng điểm khống chế ảnh cần thiết khi sử dụng UAV/RTK để xây dựng DSM đã được tiến hành trong nhiều nghiên cứu, tuy nhiên các nghiên cứu này đều thực hiện khảo sát với tính năng định vị tâm chụp ảnh theo phương thức đo động thời gian thực, chưa có nghiên cứu nào thực hiện tại các mỏ lộ thiên - nơi có địa hình phức tạp, đặc biệt là chệnh lớn hơn rất nhiều so với địa hình ở các nghiên cứu ở trên.

Khi sử dụng công nghệ bay chụp UAV/RTK, người dùng có thể lựa chọn một trong hai phương pháp là định vị tâm ảnh bằng RTK hoặc PPK (xử lý sau). Trong khi chế độ bay định vị tâm chụp ảnh bằng RTK có ưu điểm là có tọa độ tâm ảnh ngay sau khi bay chụp ở thực địa, nhược điểm của phương pháp này là đòi hỏi sự kết nối liên tục giữa trạm cơ sở (Base) hoặc hệ thống trạm Cors và máy bay. Khi sử dụng trạm Base thì độ chính xác bị ảnh hưởng khi máy bay ở khoảng cách xa với trạm Base, hoặc tín hiệu bị đứt quãng do địa vật chắn giữa máy bay và trạm Base. Ngoài ra, yêu cầu phải có tọa độ trạm Base ngay trước khi máy bay cất cánh cũng làm ảnh hưởng tới tiến độ công tác thực địa. Khi sử dụng với hệ thống trạm Cors thì phương pháp này phụ thuộc vào mật độ trạm Cors và không chủ động được trong giải quyết sự cố về truyền dẫn, cần phải có sim kết nối mạng internet và mua dịch vụ của bên cung cấp Cors, mất nhiều thời gian kết nối và khởi tạo, hơn nữa trong thực tế thường gặp sự cố khi xác định tọa độ tức thời (Trần Trung Anh, 2020). Trong khi đó, phương pháp bay chụp định vị tâm ảnh PPK lại khắc phục hoàn toàn các nhược điểm trên.

Trong nghiên cứu này, tác giả đánh giá tính hiệu quả cũng như độ chính xác của DSM khi sử dụng UAV/RTK với phương thức định vị tâm chụp ảnh xử lý sau GNSS/PPK.

\section{Phương pháp nghiên cứu}

\subsection{Phưong pháp thành lập DSM}

Mô hình số bề mặt địa hình thực nghiệm được thành lập với 2 trường hợp. Trường hợp 1 chỉ sử dụng ảnh UAV/RTK và xử lý tâm chụp ảnh theo số liệu đo PPK; trường hợp 2 sử dụng ảnh UAV/RTK, xử lý tâm chụp ảnh theo số liệu đo PPK kết hợp với điểm khống chế ảnh mặt đất.

Ở trường hợp 2, tọa độ tâm chụp ảnh được bình sai trên phần mềm RTKlib 2.4 .3 và Aerotas P4RTK PPK Adjustments V1.0. Sau khi bình sai, các tâm chụp ảnh có sai số từ đề-xi-mét trở lên được loại bỏ. Tọa độ tâm chụp ảnh có sai số cỡ 
xen-ti-mét được giữ lại tham gia vào quá trình xây dựng DSM. Các tấm ảnh được ghép tâm chính xác và xử lý trên phần mềm Agisoft Metashape Professional. Trong trường hợp này, số lượng điểm khống chế lần lượt được thay đổi là 01,02 và 03 nhằm mục đích đánh giá số lượng điểm tối thiểu mà vẫn đảm bảo độ chính xác của mô hình DSM.

Để có cơ sở đánh giá độ chính xác mô hình DSM, ngoài sử dụng DSM do mỏ thành lập bằng máy toàn đạc điện tử, nhóm nghiên cứu đã thiết kế và thành lập 50 điểm GCP trên khu vực thực nghiệm. Các điểm GCP được đánh dấu bằng tiêu khống chế ảnh có kích thước $60 \times 60 \mathrm{~cm}$ in 2 màu vàng và đen trên giấy bạt có độ phản xạ tốt (Hình 1), vị trí các điểm được bố trị tại các tầng khai thác có độ cao khác nhau và phân bố đều trên toàn bộ khu vực nghiên cứu. Tọa độ được đo nối vào mốc tọa độ giải tích 1 của mỏ, độ chính xác đạt được tương đương với đường chuyền đo vẽ cấp 2 theo TCVN ngành Trắc địa Mỏ (Bộ Công thương, 2015).

\section{2. Đánh giá độ chính xác mô hình DSM}

Độ chính xác của mô hình DSM được đánh giá bằng 2 phương pháp. Phương pháp 1 là so sánh các điểm trên mô hình với các điểm khống chế mặt đất tương ứng. Các điểm khống chế mặt đất được xác định trước tọa độ, độ cao và không tham gia vào quá trình xử lý ảnh. Cụ thể các công thức từ (1) tới (7) được sử dụng để đánh giá độ chính xác DSM:

$$
\begin{aligned}
\Delta X_{i} & =X_{D S M_{i}}-X_{G C P_{i}} \\
\Delta Y_{i} & =Y_{D S M_{i}}-Y_{G C P_{i}}
\end{aligned}
$$

$$
\begin{gathered}
\Delta H_{i}=H_{D S M_{i}}-H_{G C P_{i}} \\
R M S E_{X}=\sqrt{\left[(1 / n) \sum_{i=1}^{n} \Delta X_{i}^{2}\right]} \\
R M S E_{Y}=\sqrt{\left[(1 / n) \sum_{i=1}^{n} \Delta Y_{i}^{2}\right]} \\
R M S E_{X Y}=\sqrt{\left\{(1 / n) \sum_{i=1}^{n}\left[\Delta X_{i}^{2}+\Delta Y_{i}^{2}\right]\right\}} \\
R M S E_{H}=\sqrt{\left\{(1 / n) \sum_{i=1}^{n}\left[\Delta H_{i}^{2}\right]\right\}}
\end{gathered}
$$

Trong đó: $\Delta \mathrm{X}, \Delta \mathrm{Y}, \Delta \mathrm{H}$ - các giá trị chênh lệch các thành phần tọa độ; $\operatorname{RMSE}_{X}, \operatorname{RMSE}_{Y}, \mathrm{RMSE}_{\mathrm{XY}}$, $\mathrm{RMSE}_{\mathrm{H}}$ - tương ứng là sai số trung phương theo hướng trục $\mathrm{X}$, trục $\mathrm{Y}$, mặt bằng và độ cao; $\mathrm{n}$ - tổng số điểm khống chế ảnh dùng để kiểm tra; $X_{G C P_{i}}$ và $X_{D S M_{i}}, Y_{G C P_{i}}$ và $Y_{D S M_{i}}, H_{G C P_{i}}$ và $H_{D S M_{i}}$ - tương ứng là thành phần tọa độ theo trục $X$, trục $Y$ và trục $H$ của điểm khống chế ảnh và trên mô hình DSM.

Phương pháp 2 là so sánh trực tiếp với DSM do Công ty $\mathrm{CP}$ than Đèo Nai thành lập bằng máy toàn đạc điện tử. Phương pháp này cho cái nhìn tổng thể hơn so với phương pháp 1 khi có thể so sánh được bề mặt địa hình mỏ thành lập bằng 2
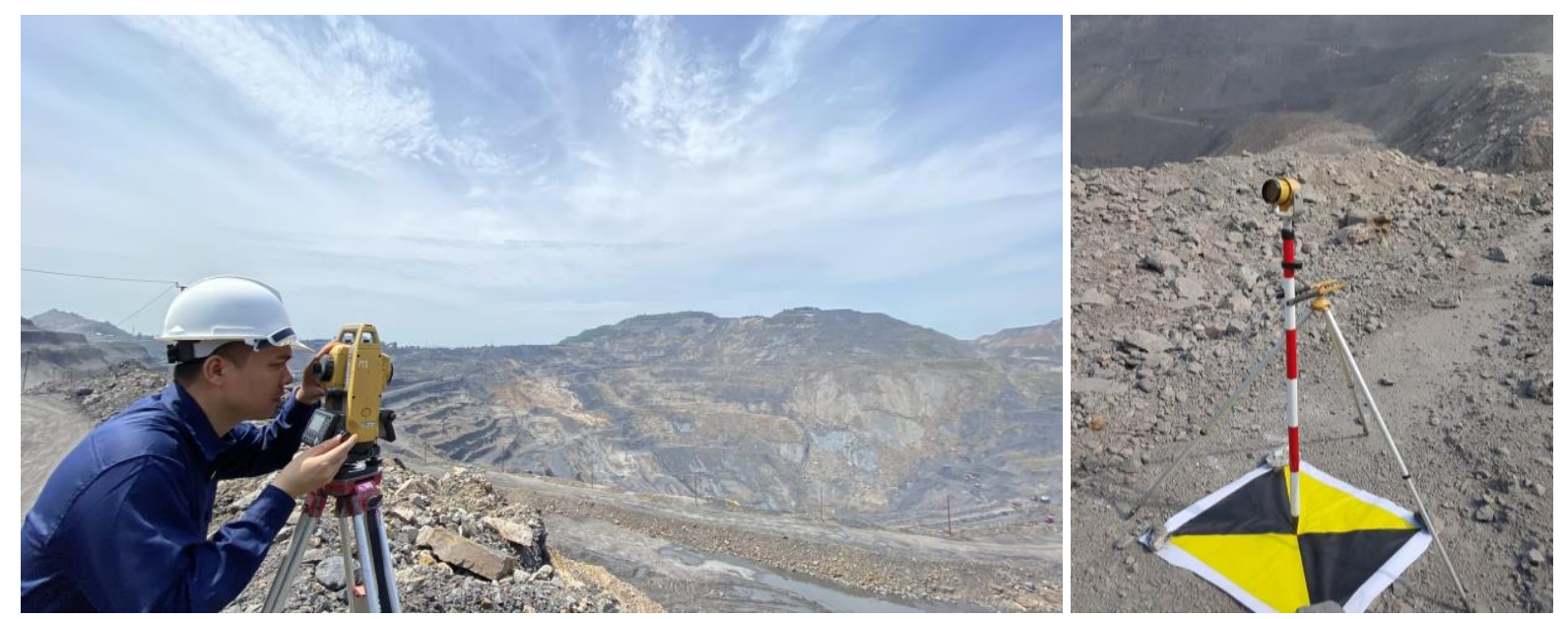

Hình 1. Đo nối tọa độ các điểm khống chế ảnh. 
công nghệ với nhau. Hai DSM được chồng xếp lên nhau, sau đó sử dụng các mặt cắt để đánh giá sự trùng khớp của hai bề mặt địa hình.

\section{Quá trình thực nghiệm}

\subsection{Thiết bị sử dụng}

Thiết bị sử dụng trong nghiên cứu là Phantom 4 RTK và máy toàn đạc điện tử Topcon ES105 (Hình 2). Phantom 4 RTK do hãng DJI sản xuất, đây là loại máy bay đang được sử dụng khá phổ biến vì giá thành thấp, nhỏ gọn và hiệu quả cao. Camera tích hợp sẵn trên máy bay có độ phân giải 20 MP, độ phân giải không gian của ảnh 2,74 $\mathrm{cm} /$ pixel khi bay ở độ cao $100 \mathrm{~m}$. Ngoài ra, phiên bản này tích hợp công nghệ định vị tâm chụp ảnh bằng GNSS động cho phép xác định tâm chụp ảnh có độ chính xác cỡ xen-ti-mét.

Các điểm chi tiết của mỏ cũng như tọa độ các điểm GCP được đo nối với hệ thống mốc khống chế cơ sở của mỏ bằng máy toàn đạc điện tử Topcon ES105, máy có thông số kỹ thuật cơ bản như sau: độ chính xác đo góc 5"; độ chính xác đo chiều dài là $2 \pm 2$ ppm.

\subsection{Bay chup UAV}

Khu vực bờ đông bắc mỏ than Đèo Nai được chọn làm khu vực nghiên cứu. Khu vực này có diện tích 70 ha, địa hình tầng bậc đặc trưng cho mỏ lộ thiên tại Quảng Ninh, chênh cao địa hình lớn nhất là $300 \mathrm{~m}$ (Hình 3 , Hình 9).

Tâm chụp ảnh được định vị theo phương thức GNSS/PPK, trong suốt quá trình bay chụp rover trên máy bay không kết nối tín hiệu với trạm Base đặt trên mặt đất (Hình 4). Trạm Base được cài đặt ở chế độ đo tĩnh với tần suất ghi tín hiệu là $5 \mathrm{~Hz}$ và thu tín hiệu đồng với rover trên máy bay.

Các thông số bay chụp được thiết kế và thực hiện trên phần mềm bản quyền DJI GS Pro, bao gồm: độ cao bay chụp $100 \mathrm{~m}$, độ phủ ngang và dọc
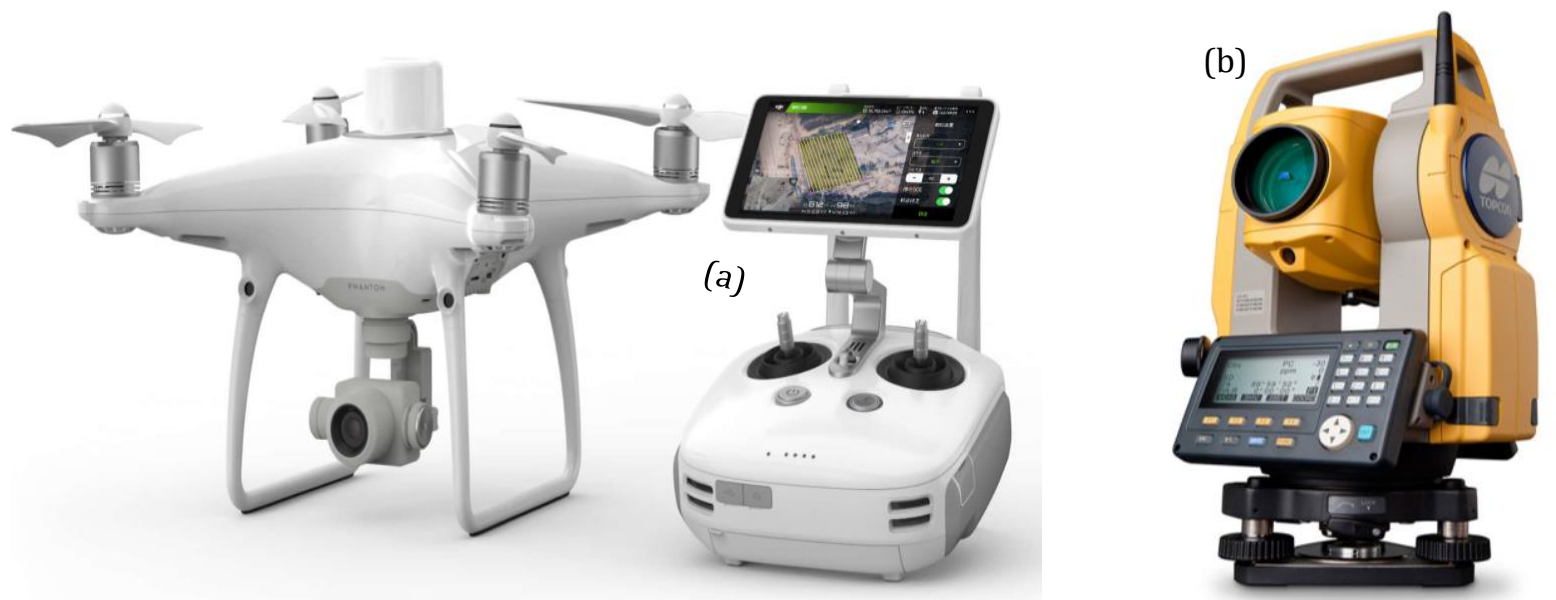

Hình 2. (a) Máy bay Phantom 4 RTK (https://www.dji.com, 2021); (b) máy toàn đạc điện tử Topcon ES105 (https://rtkvn.vn, 2021).

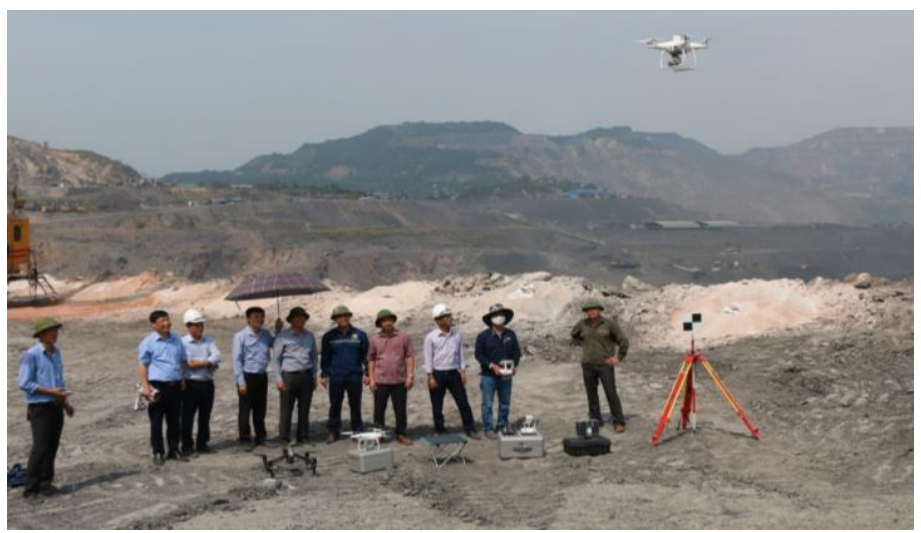

Hình 3. Bay chụp UAV trên thực địa.

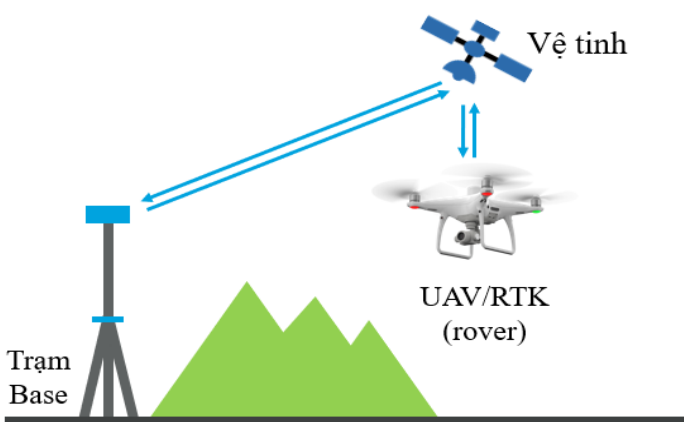

Hình 4. Nguyên lý xác định tâm chụp ảnh bằng GNSS/PPK (ảnh hiệu chỉnh tù̀ https:// grupoacre.com. 
$75 \%$. Số ca bay là 5 với tổng số ảnh thu được là 894 ảnh, độ phân giải ảnh là 2,74 cm/pixcel.

\section{Xác định tọa độ tâm chụp ảnh}

Tọa độ tâm chụp ảnh được xác định bằng phần mềm RTKlib (Hình 5), dữ liệu đầu vào bao gồm các file RINEX của trạm Base và $U A V / R T K$ và lịch vệ tinh do NASA cung cấp. Quy trình xử lý tâm chụp ảnh như Hình 6 .

\section{Kết quả và thảo luận}

Ảnh chụp từ UAV/RTK được xử lý trên phần mềm Agisoft Metashape Professional. Trường hợp không xử lý tâm chụp ảnh thì sai số tâm chụp ảnh lớn nhất là $4 \mathrm{~m}$ (Hình 7a); sai số này được cải thiện đáng kể khi các bức ảnh được gắn tọa độ tâm chụp ảnh tính từ dữ liệu định vị PPK, với sai số lớn nhất là $12 \mathrm{~cm}$ (Hình $7 \mathrm{~b}$ ). Từ đó cho thấy, việc tích hợp thêm định vị tâm chụp ảnh bằng công nghệ GNSS động cho vị trí tâm chụp ảnh bay chụp UAV có độ chính xác cao hơn so với UAV thông thường.

Thành lập mô hình DSM với các trường hợp PPK, PPK + 01 GCP, PPK+ 02 GCP và PPK+ 03 GCP. Tổng số điểm dùng để làm điểm khống chế ảnh và kiểm tra là 46 điểm. Trường hợp chỉ dùng 01 điểm khống chế ảnh là điểm A24 nằm tại trung tâm của mỏ và ở độ cao trung bình của khu vực đo vẽ, số điểm dùng để đánh giá DSM là 45 điểm; trường hợp dùng 02 điểm GCP nằm chéo nhau ở rìa của
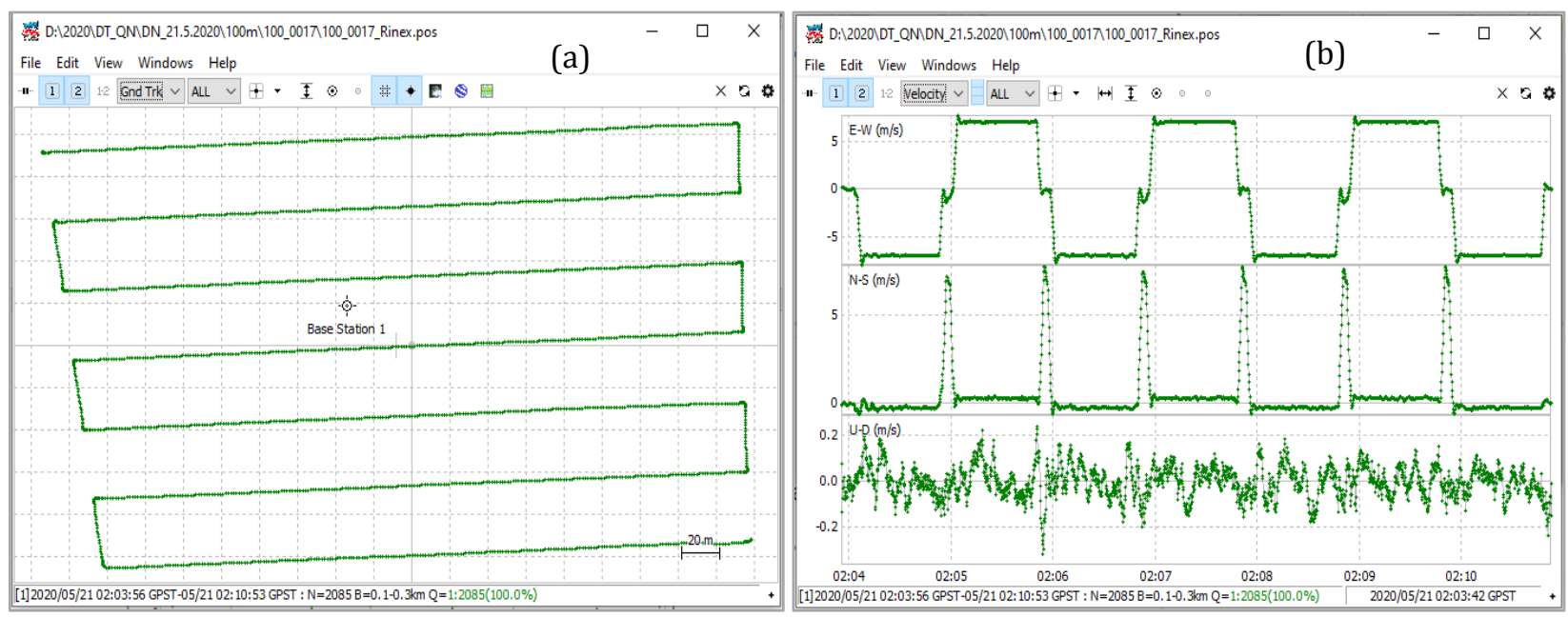

Hình 5. Vị trí tâm chụp ảnh với lời giải fix hiển thị trên phần mềm RTKlib 2.43.

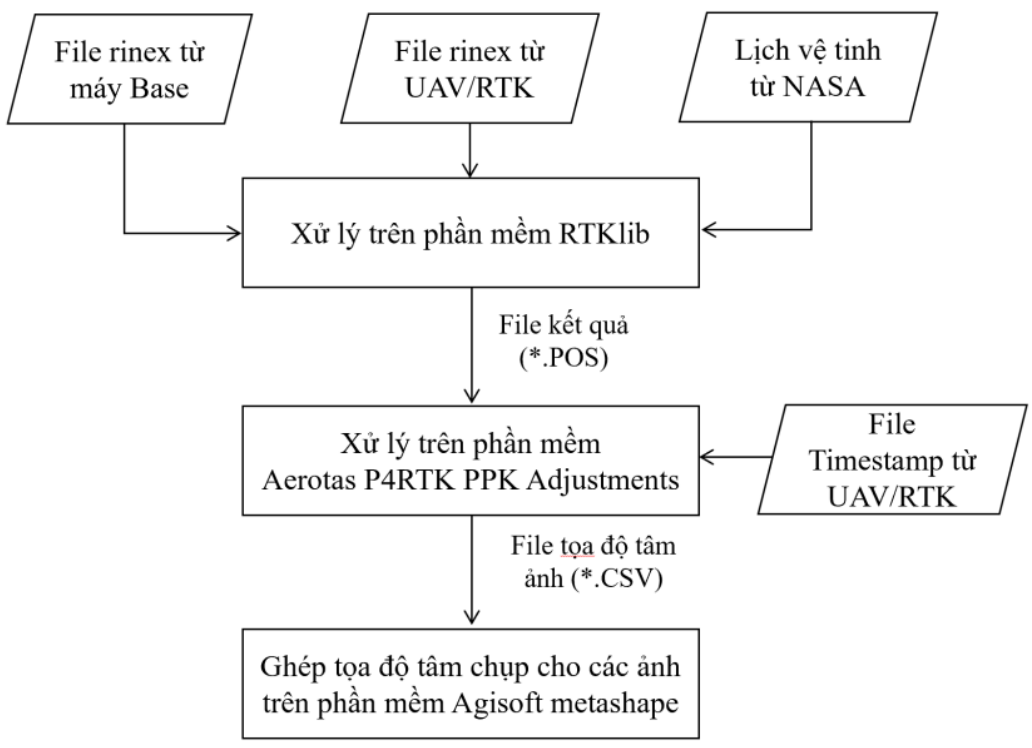

Hình 6. Qui trình xử lý tâm chụp ảnh cho các ảnh chụp bằng UAV/PPK. 


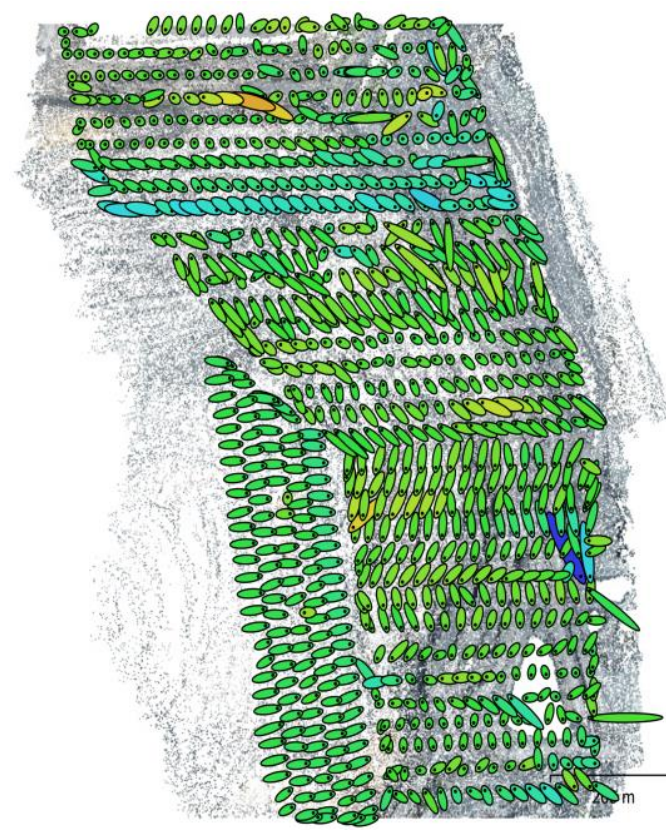

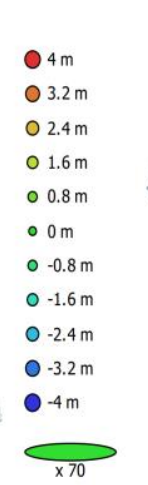

(a)

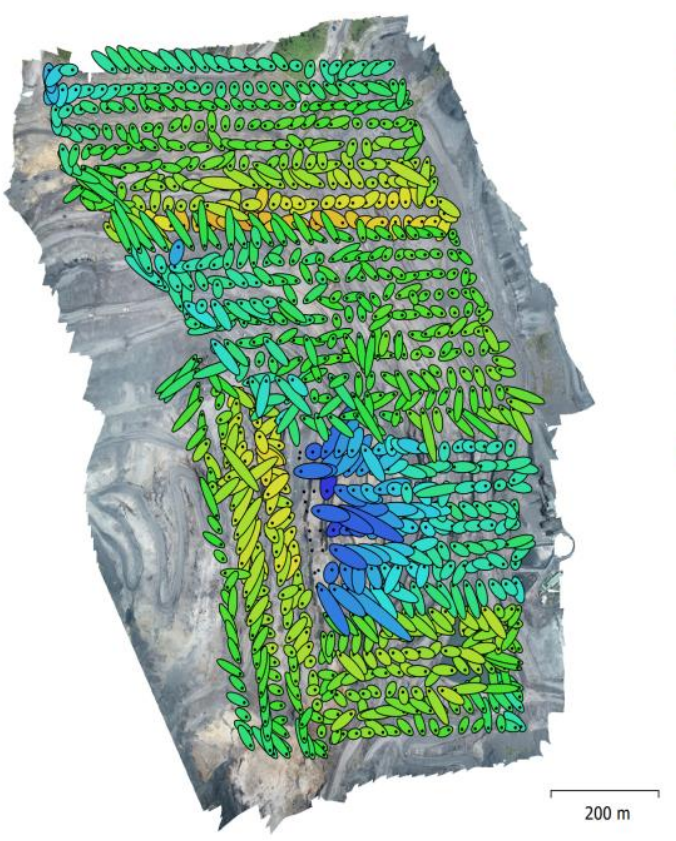

$9.6 \mathrm{~cm}$

$7.2 \mathrm{~cm}$

○ $4.8 \mathrm{~cm}$

○ $2.4 \mathrm{~cm}$

- $0 \mathrm{~cm}$

- $-2.4 \mathrm{~cm}$

$-4.8 \mathrm{~cm}$

$-7.2 \mathrm{~cm}$

$-9.6 \mathrm{~cm}$

$-12 \mathrm{~cm}$

$\times 1000$

(b)

Hình 7. Vị trí tâm chụp ảnh và elip sai số tương úng.

(a) Không xử lý tâm chụp ảnh; (b) Xử lý tâm chụp ảnh theo dũ liệu PPK.

mỏ là $\mathrm{A} 23$ và $\mathrm{A} 34$ thì số điểm kiểm tra là 44; trường hợp dùng 03 điểm GCP nằm ở 3 góc khu vực đo vẽ là $A 11, A 20$ và $A 34$ thì số điểm kiểm tra là 43. Cả trường hợp 2 và 3 thì các điểm khống chế ảnh nằm ở trên các khu vực có độ cao khác nhau. Các DSM này được đánh giá độ chính xác thông qua so sánh với các điểm GCP, các điểm này trên mô hình có độ lớn của sai số được thể hiện bằng elip sai số và màu sắc như Hình 8 . Có thể thấy rằng sai số mô hình tăng dần về phía các khu vực xa điểm GCP hoặc xa đường nối giữa 2 điểm GCP (Hình 8a,b,c,d).

Sai số của các DSM trong 4 trường hợp được trình bày tại Bảng 1 .

Bảng 1. Sai số của DSM đánh giá thông qua các điểm GCP.

\begin{tabular}{|c|c|c|c|c|c|}
\hline \multicolumn{2}{|c|}{ Trường hợp } & PPK & $\begin{array}{c}\mathrm{PPK}+1 \\
\mathrm{GCP}\end{array}$ & \multirow{2}{*}{$\begin{array}{c}\mathrm{PPK}+2 \\
\mathrm{GCP} \\
3,7\end{array}$} & \multirow{2}{*}{\begin{tabular}{|c}
$\begin{array}{c}\mathrm{PPK}+3 \\
\mathrm{GCP}\end{array}$ \\
4,0 \\
\end{tabular}} \\
\hline & $\mathrm{RMSE}_{\mathrm{X}}$ & 5,1 & 4,7 & & \\
\hline \multirow{3}{*}{$\begin{array}{c}\text { phương } \\
(\mathrm{cm})\end{array}$} & \begin{tabular}{|l|}
$\mathrm{RMSE}_{\mathrm{Y}}$ \\
\end{tabular} & 4,5 & 4,2 & 2,5 & 2,3 \\
\hline & RMSE $_{\mathrm{XI}}$ & 6,8 & 6,3 & 4,5 & 4,6 \\
\hline & $\mathrm{RMSE}_{\mathrm{H}}$ & 34,3 & 7,3 & 4,7 & 3,7 \\
\hline \multirow{4}{*}{$\begin{array}{l}\text { Sai số vị trí } \\
\text { điểm kiểm } \\
\text { tra yếu } \\
\text { nhất }(\mathrm{cm})\end{array}$} & $\Delta \mathrm{Xmax}$ & 6,3 & 4,6 & 7,6 & 7,8 \\
\hline & $\Delta Y \max$ & 1,4 & 4,3 & $-3,1$ & $-3,9$ \\
\hline & $\Delta \mathrm{XYmax}$ & 6,4 & 6,3 & 8,2 & 8,7 \\
\hline & $\Delta \mathrm{Hmax}$ & $-54,7$ & $-14,2$ & $-5,9$ & $-5,2$ \\
\hline
\end{tabular}

Nhận thấy rằng, khi sử dụng ảnh bay chụp UAV/RTK và không dùng bất kỳ điểm khống chế ảnh nào thì DSM có sai số trung phương vị trí mặt bằng cỡ xen-ti-mét $\left(\mathrm{RMSE}_{\mathrm{XY}}=6,8 \mathrm{~cm}\right)$, sai số này hầu như không được cải thiện khi dùng 01 điểm khống chế ảnh mặt đất (RMSE $\left.\mathrm{XY}_{\mathrm{XY}}=6,3 \mathrm{~cm}\right)$. Qua đây, có thể thấy rằng: khi sử dụng UAV/RTK cho mô hình DSM có độ chính xác vị trí mặt bằng đáp ứng được yêu cầu thành lập bản đồ địa hình tỷ lệ lớn $(1: 500 \div 1: 2000)$ tại các mỏ lộ thiên mặc dù không dùng bất kỳ điểm GCP nào. Cũng từ Bảng 1 cho thấy, ngược lại với thành phần tọa độ mặt bằng, độ cao của các mô hình số bề mặt có lại sai số lớn, trường hợp chỉ sử dụng tâm chụp ảnh PPK sai số trung phương trung bình độ cao của DSM là $\mathrm{RMSE}_{\mathrm{H}}=34,3 \mathrm{~cm}$, sai đó độ cao điểm yếu nhất trên mô hình này là $-54,7 \mathrm{~cm}$, đối chiếu theo qui phạm không thể sử dụng các mô hình này để thành lập bản đồ tỷ lệ lớn ở mỏ lộ thiên. Sai số này được cải thiện $75 \%$ khi sử dụng 1 điểm khống chế ảnh (PPK+1 GCP), với sai số trung phương trung bình độ cao của DSM và sai số vị trị điểm yếu nhất lần lượt là $7,3 \mathrm{~cm}$ và $-14,2 \mathrm{~cm}$. Khi sử dụng 2 điểm GCP sai số chỉ còn $4,7 \mathrm{~cm}$ tính trung bình cho toàn mô hình DSM và vị trí điểm yếu nhất sai số $-5,9 \mathrm{~cm}$. Sai số $\mathrm{RMSE}_{\mathrm{H}}=3,7 \mathrm{~cm}$ và $\Delta \mathrm{Hmax}=-5,2 \mathrm{~cm}$ khi sứ dụng 03 điểm GCP, so với trường hợp sử dụng 02 điểm GCP thì sai số được cải thiện không nhiều. 

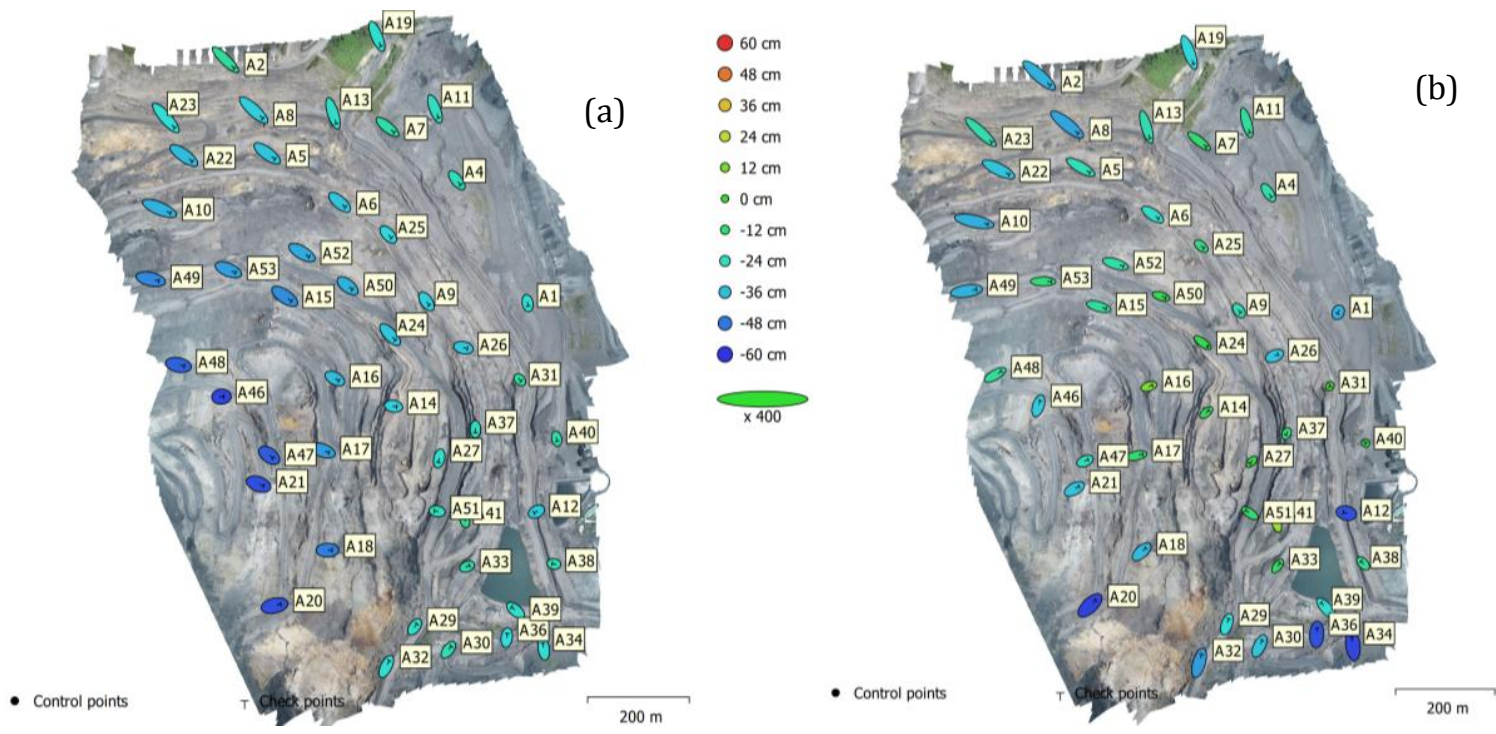

$$
\begin{aligned}
& \text { - } 15 \mathrm{~cm} \\
& \text { - } 12 \mathrm{~cm} \\
& \text { O } 9 \mathrm{~cm} \\
& \text { - } 6 \mathrm{~cm} \\
& \text { - } 3 \mathrm{~cm} \\
& \text { - } 0 \mathrm{~cm} \\
& \text { - }-3 \mathrm{~cm} \\
& \text { - }-6 \mathrm{~cm} \\
& \text { - }-9 \mathrm{~cm} \\
& -12 \mathrm{~cm} \\
& -15 \mathrm{~cm} \\
& \hline
\end{aligned}
$$
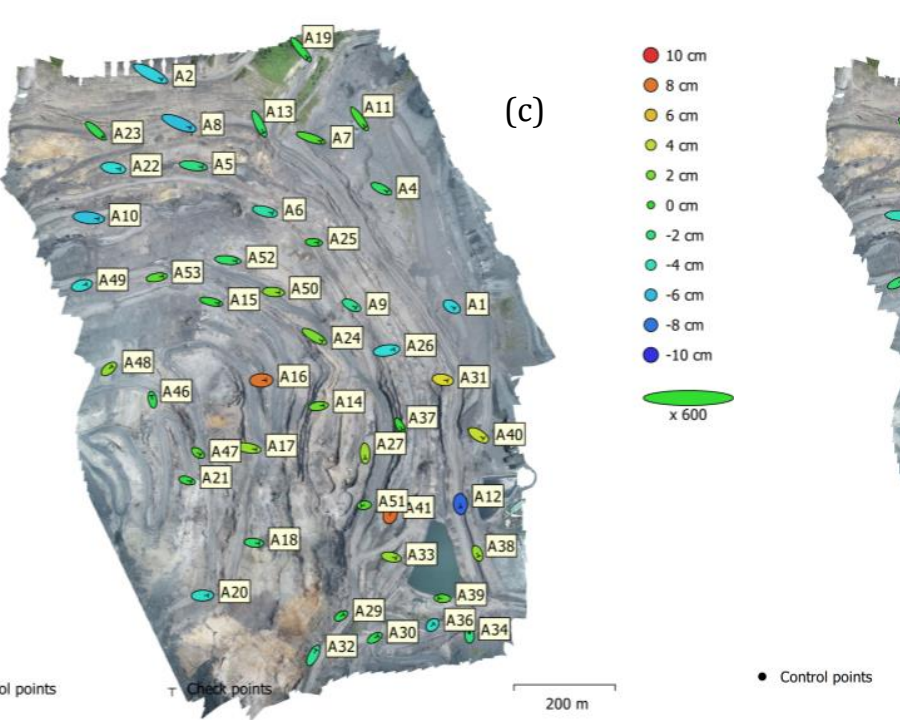

(d)

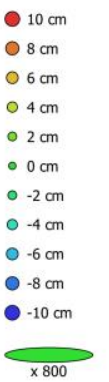

Hình 8. Vị trí các điểm GCP và elip sai số.

(a) $P P K$, (b) $P P K+01 G C P$, (c) $P P K+02 G C P$, (d) $P P K+03 G C P$.

DSM thành lập từ 4 trường hợp trên cũng được lần lượt chồng ghép lên DSM do Công ty $\mathrm{CP}$ than Đèo Nai thành lập cùng thời điểm bằng thiết bị toàn đạc điện tử. Sử dụng các mặt cắt địa hình theo các hướng khác nhau để xác định sự trùng nhau của 2 bề mặt

Từ các mặt cắt địa hình cho thấy trường hợp không sử dụng điểm khống chế ảnh mặt đất (trường hợp 1) thì 2 đường bề mặt địa hình cách xa nhau cỡ $30 \div 50 \mathrm{~cm}$, trường hợp 2 cỡ $10 \div 20 \mathrm{~cm}$. Trường hợp 3 và 4 cho kết quả tốt hơn hẳn và tương đồng nhau với độ lệch giữa 2 đường địa hình cỡ $3 \div 9 \mathrm{~cm}$. Hình 9 thể hiện sự trùng nhau giữa DSM trường hợp sử dụng 2 GCP so với DSM do mỏ Đèo Nai thành lập. Từ đó, có thể khẳng định DSM thành lập bằng công nghệ UAV/RTK, xác định tâm chụp ảnh bằng $\mathrm{PPK}$ và sử dụng 02 điểm GCP trở lên đạt độ chính xác cao, có thể dùng để biên tập bản đồ địa hình tỷ lệ lớn cho mỏ lộ thiên.

\section{Kết luận}

Độ chính xác của các DSM được đánh giá thông qua 2 phương pháp: (1) Sử dụng các điểm GCP được xác định trước tọa độ và độ cao; (2) So sánh trực tiếp với DSM do Công ty CP than Đèo Nai thành lập bằng máy toàn đạc điện tử. Kết quả nghiên cứu đã rút ra các kết luận sau đây: 

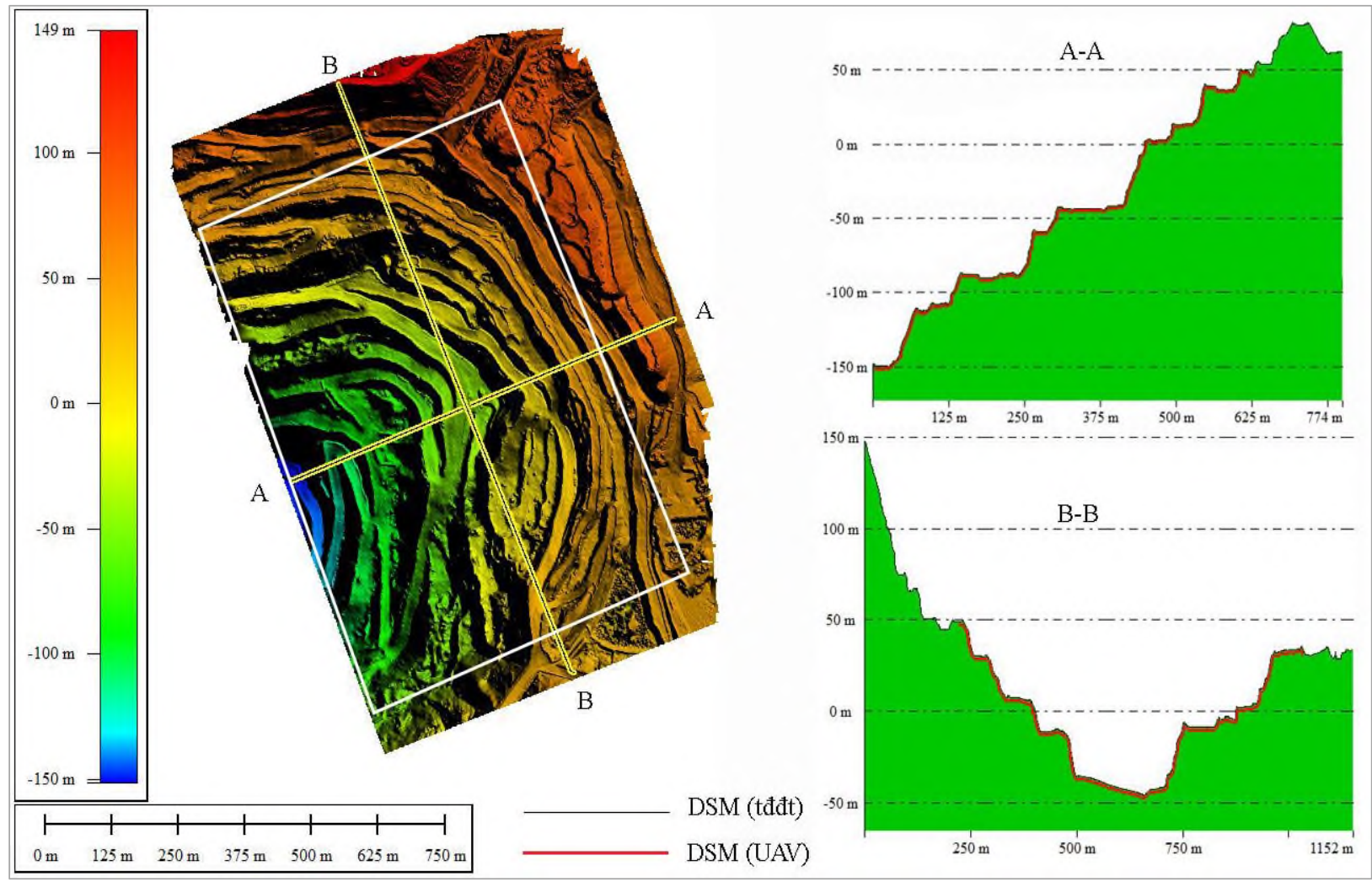

Hình 9. So sánh mô hình số địa hình thành lập bởi UAV và toàn đạc điện tử.

Khi sử dụng ảnh UAV/RTK và không dùng điểm khống chế ảnh mặt đất, DSM được thành lập với độ chính xác mặt bằng đạt yêu cầu thành lập bản đồ địa hình tỷ lệ lớn theo Qui phạm Trắc địa mỏ, tuy nhiên độ cao có sai số vượt hạn sai cho phép. Sai số độ cao của DSM thành lập từ ảnh bay chụp bằng UAV/RTK được cải thiện đáng kể khi sử dụng 01 điểm khống chế ảnh mặt đất và sai số này đạt xen-ti-mét khi sử dụng 02 điểm khống chế ảnh mặt đất.

Khi tăng số lượng điểm khống chế ảnh mặt đất lên 03 điểm thì độ chính xác của DSM khá tương đồng như khi sử dụng 02 điểm khống chế. Có thể khẳng định với diện tích khoảng 70 ha và điều kiện địa hình biến đổi lớn của mỏ lộ thiên thì chỉ cần 02 điểm khống chế mặt đất là đảm bảo độ chính xác thành lập bản đồ tỷ lệ lớn 1:500.

Cần tiếp tục khảo sát công nghệ này với các diện tích khác nhau, tại các dạng địa hình mỏ khác trong Tập đoàn Công nghiệp Than - Khoáng sản Việt Nam để xác định được số lượng điểm khống chế ảnh mặt đất tối ưu nhằm vừa đảm bảo về độ chính xác thành lập bản đồ, vừa giảm thiếu khối lượng đo ngoại nghiệp.

\section{Đóng góp của các tác giả}

Tác giả đóng góp 100\% nội dung của bài báo.

\section{Tài liệu tham khảo}

Bộ Công Thương. (2015). Tiêu chuẩn Việt Nam ngành Trắc Địa Mỏ. Viện tiêu chuẩn quốc gia Việt Nam, Hà Nội.

Bui, D.T., Long, N. Q., Xuan - Nam, B., Viet Nghia, N., Chung, P.V., Canh, L.V., Phuong Thao, T.N., Dung, B.T., Kristoffersen, B. (2017). Lightweight unmanned aerial vehicle and structure - from motion photogrammetry for generating digital surface model for open - pit coal mine area and its accuracy assessment. In International Conference on Geo - Spatial Technologies and Earth Resources. Springer, 17 - 33. DOI: 10.1007/978-3-319-68240-2_2.

Bui, N. Q., Le, D. H., Nguyen, Q. L., Tong, S. S., Duong, A. Q., Pham, V.H., Phan, T. H., Pham, T. L. (2020). Method of defining the parameters for UAV point cloud classification algorithm. Journal of 
the Polish Mineral Engineering Society, 46(1), 49 - 56, 2020. DOI 10.29227/IM-2020-02-08.

DJI (2020). Phantom 4 RTK Visionary Intelligence, https://www.dji.com/phantom-4-rtk.

Fazeli, H., Samadzadegan, F., Dadrass Javan, F. (2016). Evaluating the potential of RTK - UAV for automatic point cloud generation in 3D rapid mapping. ISPRS - International Archives of the Photogrammetry, Remote Sensing and Spatial Information Sciences, XLI - B6, 221 - 226. DOI:10.5194/isprsarchives-XLI-B6-221-2016.

Forlani, G., Dall'Asta, E., Diotri, F., Cella, U., Roncella, R., Santise, M. (2018). Quality assessment of DSMs produced from UAV flights georeferenced with on - board RTK positioning. Remote Sensing, 10(2), 1 - 22. DOI: $10.3390 / \mathrm{rs} 10020311$

Long, N. Q., Xuan - Nam, B., Cuong C. X., Canh, L. V. (2019). An approach of mapping quarries in Vietnam using low - cost Unmanned Aerial Vehicles. Sustainable Development of Mountain Territories, 11(2), 199 - 210. DOI: 10.21177/ 1998-4502-2019-11-2-199-201.

Nguyen, Q. L., Le, T. T. H., Tong, S. S., Kim, T. T. H (2020). UAV Photogrammetry-Based For Open Pit Coal Mine Large Scale Mapping, Case Studies In Cam Pha City, Vietnam. Sustainable Development of Mountain Territories, 12(4), 501-509. DOI: 10.21177/1998-4502-2020-12-4501-509.

Nguyen, Q. L., Ropesh, G., Bui, K. L., Le, V. C., Cao, X. C., Pham, V. C., Bui, N. Q., Xuan - Nam, B. (2020). Influence of Flight Height on The Accuracy of UAV Derived Digital Elevation Model at Complex Terrain. Inżynieria Mineralna, 45(1), p. 179 - 186. DOI: http:// doi.org/10.29227/IM- 2020-01-27.

Nguyen Quoc Long, Michał M Buczek, Sylwia A Szlapińska, Bui Xuan Nam, Nguyen Viet Nghia, Cao Xuan Cuong. (2018). Accuracy assessment of mine walls' surface models derived from terrestrial laser scanning. International Journal of Coal Science \& Technology, 5(3), 328 - 338, DOI: https://doi.org/10.1007/s40789-018 $-0218-1$

Nguyễn Quốc Long, Lê Văn Cảnh, (2020). Khả năng ứng dụng thiết bị bay không người lái (UAV) kinh phí thấp để đo vẽ kiểm kê trữ lượng khoáng sản mỏ lộ thiên. Công nghiệp mỏ, 02, 79 - 85.

Nguyen Viet Nghia, Nguyen Quoc Long, Pham Cong Khai, Le Van Canh, Michal Buczek. (2016). Applications of Continuously Operating Reference Station Technology for Surveying and Mapping of Open Pit Mine. International Conference on Advances in Mining and Tunneling, ICAMT (2016), 247-253.

Nguyen Viet Nghia, Nguyen Quoc Long, Nguyen Thi Cuc, Xuan-Nam Bui. (2019). Applied Terrestrial Laser Scanning for coal mine High Definition mapping. World of Mining - Surface and Underground, 71(4), 237-242.

Nguyễn Viết Nghĩa. (2020). Building DEM for deep open-pit coal mines using DJI Inspire 2 (in Vietnamese). Journal of Mining and Earth Sciences, 61(1), (Feb, 2020), 1-10. DOI: https://doi.org/10.46326/ JMES.2020.61(1). 01.

Taddia, Y., Stecchi, F., Pellegrinelli, A. (2019). Using DJI Phantom 4 RTK drone for topographic mapping of coastal areas. Int. Arch. Photogramm. Remote Sens. Spatial Inf. Sci. XLII 2/W13, 625 - 630. DOI: 10.5194/isprsarchives- XLII-2-W13-625-2019.

Trần Trung Anh, Quách Mạnh Tuấn. (2020). Phan tích lựa chọn chế độ định vị tâm chụp chính xác của máy bay không người lái trong thành lập bản đồ địa hình tỷ lệ lớn. Hội nghị toàn quốc khoa học trái đất và tài nguyên với phát triên bền vũng (ERSD 2020), 1 - 8.

Van Canh, L., Xuan Cuong, C., Quoc Long, N., Thi Thu Ha, L., Trung Anh, T., \& Bui, X. - N. (2020). Experimental Investigation on the Performance of DJI Phantom 4 RTK in the PPK Mode for 3D Mapping Open - Pit Mines. Test, 1(2), 65 - 74. https://doi.org/10.29227/IM-2020-02-10.

Zhang, H., Aldana - Jague, E., Clapuyt, F., Wilken, F., Vanacker, V., Van Oost, K., (2019). Evaluating the potential of post - processing kinematic (PPK) georeferencing for UAV - based structure - from - motion (SfM) photogrammetry and surface change detection. Earth Surface Dynamics, 7, 807 - 827. 